Danach wurden von jedem Steckling die vier obersten Blätter (gleiches Frischgewicht pro Filter) abgenommen, grob zerkleinert und mit $40 \mathrm{~cm}^{3}$ Peroxyd-freiem Äther entsprechend den Anweisungen von ${ }^{2}$ ) auf ,freie" + ,gebundene Auxine" (- Warm-Extrakt) bzw. in einer späteren Serie auf ,freie Auxine" (= Kalt-Extrakt) extrahiert. Die quantitative Bestimmung der Auxine erfolgte mit Hilfe des KW-Testes ${ }^{1}$,

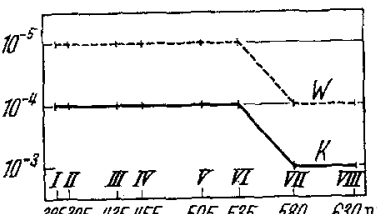

$385395435455505535 \quad 580 \quad 630 \mathrm{~nm}$

Fig. 1. Bestimmung des Auxin-Gehaltes nach dem KW-Test (s. Text), W WarmExtrakt; $K$ Kalt-Extrakt. Ordinate: Extrakt-Verdúnnung. Abszisse: Wellenlänge und benutzte Schottfilter: 1 GG $13 / 2 \mathrm{~m}$; II GG $13 / 4 \mathrm{~mm}$; III $\mathrm{GG} 3 / 4 \mathrm{~mm}$; IV $\mathrm{GG}$ $5 / 4 \mathrm{~mm} ; \mathrm{VOG} 4 / \mathrm{mm}$; VI OG $1 / 2 \mathrm{~mm}$; VII OG $3 / 1 \mathrm{~mm}$; VIII $\mathrm{RG} 2 / 2 \mathrm{~mm}$ wobei stets nur das erste.Fördetmaximum bewertet wurde. Die Mittelwerte aus 10 (Warm-Ex trakt) bzw.7 (Kalt-Extrakt) unter sich sehr einheitlichen Wiederholungsserien sind als Absolut (nicht als Differenz)-Werte in Fig. 1 dargestellt. Sie zeigen sowohl für die Kalt-als auch für die Warm-Extrakte, daß der Gehalt an freien und gebundenen Auxinen bei ausschlièßlicher Einwirkung von Licht $\lambda<730$, $>580 \mathrm{~nm}$ um eine volle Potenz niedriger liegt als bei zusätzlicher Einwirkung von kürzerwelligem Licht $>385 \mathrm{~nm}$, daß aber bei der verwendeten Methode zwischen $\lambda>385 \mathrm{~nm}$ und $<505 \mathrm{~nm}$ keine speziellen Förder bzw. Hemmbereiche festgestellt werden konnten. Eine max. Förderung der Auxin-Synthese - und nur diese sollte hier untersucht werden - haben wir also lediglich zwischen $505 \mathrm{~nm}$ und $580 \mathrm{~nm}(\sim 535 \mathrm{~nm})$ zu erwarten. Aus technischen Gründen konnte leider die Bedeatung des langwelligen Rots $(>730 \mathrm{~nm})$ nicht mit untersucht werden.

Institut für Botanik der Technischen Hochschule, Hannover

U. RUGE*

Eingegangen am 24. Mai 1960

1) HAHn, I.M.: Gartenbauwiss. 24 (6), 363 (1959). - 2) HEMBerg, T.: Physiol. Plantarum a) 7, 312 (1954); b) 11, 284 (1958)

\section{Ein Beitrag zur Prüfung der Keimungsfähigkeit} einiger Pflanzensamen

Schon längere Zeit sind einige chemische Reagenzien, die zur Prüfung der Keimungsfähigkeit verschiedener Pflanzensamen angewandt werden können, bekannt. Diese chemischen Substanzen geben mit lebendigen Samen bzw. Embryonen eine von der der toten Samen verschiedene Verfärbung. So z. B. färbt Natriumtellurat $\left(\mathrm{Na}_{2} \mathrm{TeO}_{4}\right)$ Embryonen von lebendigen Samen blau, während tote Samen nicht gefärbt werden. Indigokarmin dagegen färbt blau nur tote Samen, während lebendige Samen nicht gefärbt werden. In der letzten Zeit verwendet man $z u$ diesem Zwecke das 2,3,5-Triphenyltetrazoliumchlorid oder das entsprechende Bromid $\left.{ }^{1-3}\right)$ : Die Embryonen der lebendigen Samen werden nach vollständigem 6stündigem Befeuchten intensiv rot gefärbt. Es wird aber bloß der lebendige Teil des Embryos gefärbt, so daß nicht nur die Keimungsfähigkeit, sondern auch die Qualität der gekeimten Samen bestimmt werden kann. Die TetrazoliumSalze bestimmen auf diese Weise die Dehydrogenase-Aktivität der keimungsfähigen Samen und werden auch in der Enzymologie zur Bestimmung der Aktivität verschiedener Dehydrogenasen erfolgreich verwendet ${ }^{4-7}$ )

In unserem Iaboratorium konnte gezeigt werden, daß auch $\alpha$-Naphthylamin zur Prüfung der Keimungsfähigkeit angewandt werden kann. Die geprüften Samen werden mit gesättigter wäßriger Lösung des $\alpha$-Naphthylamins nur leicht überschichtet und dann einige Stunden lang stehen gelassen. Dabei muß aber darauf geachtet werden, daß alle Samen überschichtet werden; die Samen, die an der Oberfläche schwimmen, werden weggenommen. Unter diesen Bedingungen werden die Embryonen der toten Samen braunrot gefärbt, die lebendigen Samen werden dagegen nicht gefärbt. Die Intensitä der Färbung ist nicht zu groß, aber doch gut ersichtlich.

Auf diese Weise werden die Samen der einzelnen Getreidearten (Weizen, Roggen, Gerste) befriedigend auf die Keimungsfähigkeit geprüft. Bei dem Mais muß man aber die Samen längs zerschneiden, um die Embryonen der $\alpha$-Naphtylamin-Lösung zugänglich zu machen. Auch bei der Gerste ist das Zerschneiden der Samen zu empfehlen. Bei den Dicotyledonen werden die toten Samen sehr verschieden gefärbt; in einigen Fällen werden auch lebendige Samen gefärbt.
In jedem Fall sind aber die Färbungen der toten und der lebendigen Samen gut zu unterscheiden.

Am meisten werden die toten Samen rosa bis braun gefärbt; bei der weißen Lupine (Lupinus albus) tritt aber eine violette Färbung auf. Ein Vorteil dieser nenen Methode der Keimungsfähigkeitsprüfung beruht in der Tatsache, daß $\alpha$-Naphthylamin billiger und zugänglicher ist als die anderen Reagenzien, insbesondere die Tetrazoliumsalze.

Landreirtschaftiche Prüfungsstation, Prag 3, Karlin (Tschechoslowakei)

Eingegangen am 9. Mai 1960

J. KLEЕัNHA*)

*) Jetzige Adresse: Praha 2, Na Zderaze 11, Tschechoslowakei. 1) LAKon, G.: Ber, dtsch. bot. Ges. 60, 299, 434 (1942). 2) LAKON, G.: Plant Physiol. 24, 389 (1949). - 9) Cotrell, H. J.: Nature [London] 159, 748 (1947); Ann. Appl. Biol. 35, 123 (1948). 4) KuHN, R., u. F. LinkE: Liebigs Ann. Chem, 578, 155 (1952). 5) Jensen, C.O., W. Sacks u. F.A. Baldauski: Science 113, 65 (1951). - $\left.{ }^{6}\right)$ Kun, E.: Proc. Soc. Exp. Biol. Med. 78, 195 (1951). 7) PÁdr, Z.: Tetrazoliové solí-Státní zdravotnické nakladatelství, Praha 1959.

\section{Besteht ein Zusammenhang zwischen dem Saugkraftvermögen} von Kiefernsamen und dem Standort?

Ausgehend von der Annahme, daß sich Standortrassen von Pinus silvestris keimungsphysiologisch unterscheiden könnten, war es Aufgabe der hier mitgeteilten Untersuchungen, festzustellen, ob Samen von Einzelstämmen extrem trokkener und nasser Standorte eines Wuchsgebietes Unterschiede im Saugkraftvermögen aufweisen. Nach einer ähnlichen Methode, wie sie GAssNer und BaUmGar $\operatorname{TEN}^{1}$ ) anwendeten, kamen von 28 Kiefern (davon 14 von nassen und 14 von trokkenen Standorten) jeweils $5 \times 50$ Samen in $1 \mathrm{G} 3$-Frit-

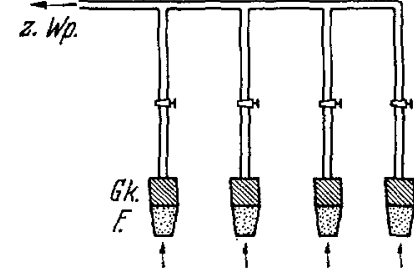

Fig. 1. Apparatur, schematisch. z.Wp. zur Wasserstrahlpumpe; $G k$. Gärkappe; $F$. Fritte ten, die mit $15 \mathrm{~cm}^{3}$ Glukoselösung von $8 \mathrm{Atm}$ osmotischen Druckes (bei $20^{\circ} \mathrm{C}$ ) gefüllt waren. Als Kontrolle diente stets die gleiche Anzahl Samen, die in Leitungswasser, im übrigen jedoch unter denselben Bedingungen, keimten. Die Fritten, mit durchbohrten Gärkappen verschlossen, waren mit einer Wasserstrahlpumpe
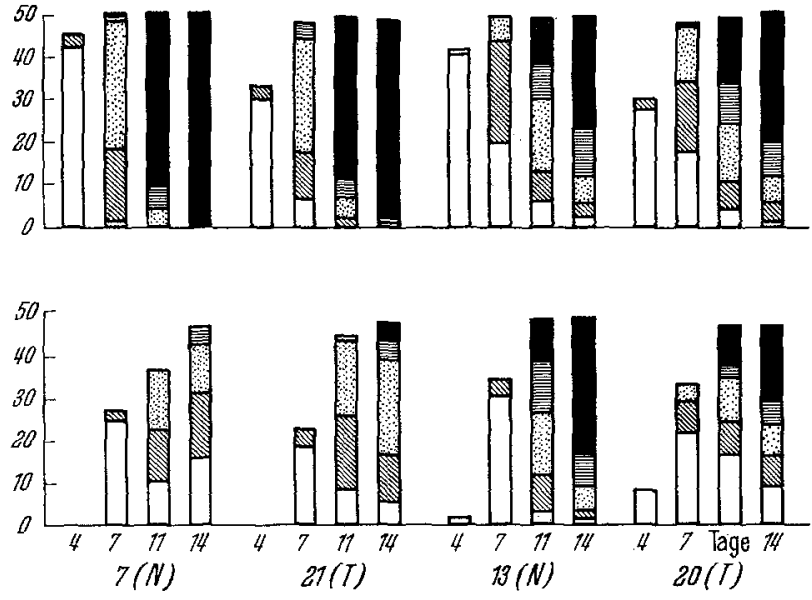

Fig. 2. Entwicklung der Keimlinge aus Samen verschiedener Bäume von nassem $(N)$ bzw. trockenem $(T)$ Standort nach 4,7 , 11 und 14 Tagen. Ordinate: Anzahl der gekeimten Samen. Obere Reihe: Fritte mit Wasser gefïllt; untere Reihe: Glukoselösung (8 Atm.). - Die Schraffuren entsprechen 5 Klassen von Keimlingsgrößen: 0 bis $2 \mathrm{~mm}$ (leer); 2 bis $5 \mathrm{~mm}$ (schräge Schraffen); 5 bis $10 \mathrm{~mm}$ (punktiert); 10 bis $15 \mathrm{~mm}$ (horizontale Schraffen); >15 mm (schwarz)

verbunden. Durch Anlegen eines geringen Unterdrucks erfolgte die ständige Belüftung (Fig. 1). Täglich wurde die Glukoselösung erneuert, um ein Verpilzen und ein Herabsinken des PH-Wertes möglichst auszuschließen. Das Keimvermögen und die Entwicklung der Keimlinge wurden nach 4, 7, 11 und 14 Tagen registriert. Keimung und Entwicklung erfolgten 